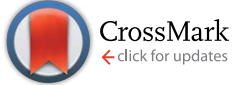

Cite this: RSC Adv., 2017, 7, 14192

Received 19th December 2016 Accepted 24th February 2017

DOI: 10.1039/c6ra28379a

rsc.li/rsc-advances

\section{Controllable synthesis and enhanced gas sensing properties of a single-crystalline $\mathrm{WO}_{3}-\mathrm{rGO}$ porous nanocomposite $\uparrow$}

\author{
Qin Hao, ${ }^{a}$ Tie Liu, ${ }^{a}$ Jingyuan Liu, ${ }^{* a}$ Qi Liu, ${ }^{a}$ Xiaoyan Jing, ${ }^{a}$ Hongquan Zhang, \\ Guoqing Huang ${ }^{c}$ and Jun Wang*a
}

In this paper, we report on a facile hydrothermal approach combined with a subsequent annealing process for the controllable synthesis of a single-crystalline $\mathrm{WO}_{3}-\mathrm{rGO}$ porous nanocomposite. The crystal structure, morphology and chemical composition of the as-obtained product were well-characterized by $\mathrm{X}$-ray diffraction, transmission electron microscopy, X-ray photoelectron spectroscopy, Fourier transform infrared spectroscopy and Brunauer-Emmett-Teller analysis. The results indicate that this hybrid structure is composed of single-crystal $\mathrm{WO}_{3}$ porous nanoflakes with a size of $500 \times 500 \mathrm{~nm}^{2}$ growing through or anchoring into a sheet-like rGO matrix. We explore the sensing performance of the gas sensor based on the as-synthesized product. Impressively, gas testing shows that the $\mathrm{WO}_{3}-\mathrm{rGO}$ nanocomposite exhibits an excellent kinetic response speed and good sensitivity toward $\mathrm{NO}_{2}$ and some volatile organic compound pollutants at a low temperature $\left(90^{\circ} \mathrm{C}\right)$. The pseudo $3-\mathrm{D}$ structure provides many channels for gas diffusion and clearly enhances sensing properties. As such, this graphene-based composite shows promising potential as a high-performance gas sensing material for real-time gas detection.

\section{Introduction}

Research studies of promising and high-quality functional materials play a significant and continuing role in material science and chemical engineering domains. ${ }^{\mathbf{1 - 4}}$ In recent years, research interest has focused on the development of selfassembled graphene-related materials with unique nano/ microstructures and these structures are expected to provide more handles to tailor the properties for different applications, such as gas sensors, ${ }^{\mathbf{1 , 4 - 6}}$ catalysis, ${ }^{7}$ lithium ion batteries ${ }^{\mathbf{8}, \mathbf{9}}$ and pseudocapacitive materials. ${ }^{\mathbf{1 0 - 1 3}}$ Graphene-related materials have stood out in the gas sensing field and attracted increasing attention for the detection of pollutants, and toxic and combustible gases at relatively low temperature. ${ }^{14-16}$ These lowcost materials exhibit excellent electrical conductivity, chemical stability, large accessible surface areas and environmental friendliness. As reported, graphene and reduced graphene oxide (rGO) have been considered as promising candidates for gas detection; numerous studies have been launched into their gas sensitive properties. ${ }^{\mathbf{1 4 , 1 5}}$ However, during synthesis, studies

${ }^{a}$ Key Laboratory of Superlight Material and Surface Technology, Ministry of Education, Harbin Engineering University, 150001, PR China. E-mail: zhqw1888@sohu.com; Fax: +864518253 3026; Tel: +8645182533026

${ }^{b}$ School of Automation, Harbin Engineering University, 150001, PR China ${ }^{c}$ Handan Purification Equipment Research Institute, Handan 056027, P. R. China

$\dagger$ Electronic supplementary information (ESI) available. See DOI: 10.1039/c6ra28379a indicate that these thin graphene sheet-like structures often undergo aggregation or restacking that is driven by minimum surface energy, which is detrimental to the enhancement of properties. Therefore, studies have focused on avoiding the aggregation of graphene sheets to achieve the fabrication of high-quality material with desirable properties. ${ }^{\mathbf{1 6 - 2 0}}$

In recent literatures, hybrid nano/micro-structures are confirmed as very effective in preventing the aggregation of graphene sheets, thus advancing progress in improving gas sensors. For instance, Sun et al. used a solution-heated method to synthesize reduced graphene oxide-based macroporous films. This rGO-oxide composite of porous films exhibited much better sensing performance than those of the corresponding pure oxide films in detecting ethanol gas. ${ }^{5}$ Also, Zhang et al. synthesized rGO-SnO ${ }_{2}$ heterojunction aerogels via a simple sol-gel method. The test results indicated that the formation of a heterojunction structure between $\mathrm{SnO}_{2}$ and rGO enhanced gas sensing activity to some VOCs gases, especially to phenol. ${ }^{21}$ Similarly, Xiao et al. obtained $\mathrm{SnO}_{2}$ nanoparticles decorated graphene composite, the gas tests showing that this composite exhibited a good sensing activity and response kinetics toward hydrogen. ${ }^{22}$ Clearly, there is a great potential in gas sensors and the properties of graphenebased gas sensing materials should be further enhanced for large-scale application.

However, the current semiconductor oxide-rGO based gas sensors still contain many limitations for practical application, especially at working temperature and response speed. ${ }^{18,21,23}$ The parameters mentioned above are crucial for the evaluation of gas 
sensors. ${ }^{24}$ And research indicated that there would be substantially difference in the sensing properties, while the chemical composition maintained but the morphologies changed, even a small variation in surface feature or crystal structure is taken place. $^{25-28}$ Therefore, at the current stage, the main emphasis in studies should be devoted to the synthesis of multifarious hybrid sensing materials with appropriate hetero-structure, optimization of composition and morphology, so as to improve the material's real-time gas detection performance at ppm, subppm, even ppb level. ${ }^{14,17,19,20}$ Tungsten(vI) trioxide $\left(\mathrm{WO}_{3}\right)$ represents an important and application-wide class of semiconductor oxides, and its micro-structure can be easily controlled by changing the solvents or additives, according to recent reports. ${ }^{25-27}$ There are many cases of interest in which $\mathrm{WO}_{3}$ is used as a major functional component of sensing materials for the detection of gases, such as VOCs, $\mathrm{NO}_{2}$ and $\mathrm{H}_{2} \mathrm{~S}^{.6-29}$ To the best of our knowledge, although scholars have reported on the synthesis of graphene-related nanocomposite, there is little attention given to the details of preparing a $\mathrm{WO}_{3}-\mathrm{rGO}$ composite and its application as a gas sensing material. . $2,27,29,30^{2}$

In this work, reduced graphene oxide based nanocomposite $\left(\mathrm{WO}_{3}-\mathrm{rGO}\right.$ ) has been successfully prepared by adopting a facile and eco-friendly hydrothermal process combining a postcalcination treatment. The synthetic route follows a typical pathway to obtain high-quality composite with hierarchical architecture. Note that this method requires a high concentration of GO precursor and introduces a small amount of $\mathrm{W}$ into the reaction system to prevent GO stacking. A series of techniques was used to characterize the morphology and analyse the chemical composition of as-obtained products. To investigate the potential application, gas sensors based on as-synthesized $\mathrm{WO}_{3}-\mathrm{rGO}$ composite (called "S1"), pure $\mathrm{WO}_{3}$ lamellas (S2, control group) and the original GO nanosheets (S3, control group) were fabricated and subsequently investigated for gas sensing performances. Interestingly, the $\mathrm{WO}_{3}-\mathrm{rGO}$ nanocomposite can be used as an effective sensing material for detection of $\mathrm{NO}_{2}$ at a low operating temperature $\left(90^{\circ} \mathrm{C}\right)$. We also found that $\mathrm{WO}_{3}-\mathrm{rGO}$ nanocomposite exhibited much better response than control groups. We describe and discuss these in detail below.

\section{Experimental section}

\subsection{Materials}

The graphite powder was purchased from Sinopharm chemical reagent Co. Ltd. Before using, the graphite powder of size 300400 mesh was sieved out. Other chemical reagents were of analytical grade and used directly after commercial purchase without further purification, unless otherwise noted.

\subsection{Synthesis of graphene oxide}

Graphene oxide (GO) was prepared from natural graphite powder through a modified Hummers' method, which is presented briefly as follows: $:^{\mathbf{8 1 0 , 1 4}}$ in a typical synthesis, $5 \mathrm{~g}$ of graphite powder was added into $100 \mathrm{~mL}$ of $\mathrm{H}_{2} \mathrm{SO}_{4}(98 \%)$ under an ice bath atmosphere $\left(0 \pm 5{ }^{\circ} \mathrm{C}\right)$ and stirred for $2 \mathrm{~h}$.
Subsequently, $30 \mathrm{~g} \mathrm{KMnO}_{4}$ was slowly added into the solution and also kept below $5{ }^{\circ} \mathrm{C}$ by an ice bath. Next, the mixture was transferred into a water bath atmosphere and stirred for $45 \mathrm{~min}$ at $50 \pm 5{ }^{\circ} \mathrm{C}$. After that, $300 \mathrm{~mL}$ of distilled water was added into the brown mixture which was then heated to $90{ }^{\circ} \mathrm{C}$ with stirring for $15 \mathrm{~min}$. To remove excess $\mathrm{KMnO}_{4}, 40 \mathrm{~mL}$ of $\mathrm{H}_{2} \mathrm{O}_{2}(5 \%)$ and $200 \mathrm{~mL}$ distilled water were poured into the mixture. The colour of the mixed solution turned to golden yellow. Finally, the asprepared GO was washed with $3 \mathrm{wt} \% \mathrm{HCl}$ and deionized water until the $\mathrm{pH} \approx 7$, then dispersed into individual sheets in distilled water at a concentration of $1 \mathrm{mg} \mathrm{mL}^{-1}$ with the aid of ultrasound, for further use.

\subsection{In situ synthesis of $\mathrm{WO}_{3}-\mathrm{rGO}$ nanocomposite}

Based on the fact that the dry GO sheets were difficult to disperse evenly in deionized water, we used a pre-obtained GO solution to prepare the target $\mathrm{WO}_{3}-\mathrm{rGO}$ nanocomposite in the following one-pot process. Typically, GO solution was ultrasonically dispersed in $60 \mathrm{~mL}$ distilled water (DW) and transferred to a boiling 3-neck-flask. Next, $1 \mathrm{mmol}$ sodium tungstate hydrate $\left(\mathrm{Na}_{2} \mathrm{WO}_{4} \cdot 2 \mathrm{H}_{2} \mathrm{O}, 99.5 \%\right.$ purity) and sodium oxalate $\left(\mathrm{Na}_{2} \mathrm{C}_{2} \mathrm{O}_{4} \cdot 2 \mathrm{H}_{2} \mathrm{O}, 99.5 \%\right.$ purity) were added and dissolved ultrasonically into the above solution. In brief, a moderate amount of acidic solution ( $2 \mathrm{M} \mathrm{HCl}$ ) was added dropwise at a controlled rate ( $c a .0 .1 \mathrm{~mL}$ per minute) by a microsyringe under vigorous stirring until the $\mathrm{pH}$ value of the whole solution was adjusted to 2 . Then, the above mixture was transferred into a Teflon-lined stainless-steel autoclave and kept at $160{ }^{\circ} \mathrm{C}$ for $10 \mathrm{~h}$. After cooling down naturally, the solid-precursor material was collected by symmetric centrifugation and washed several times with ethanol and distilled water in turn to remove excess ions. After freeze drying, the sample was calcined at $400{ }^{\circ} \mathrm{C}$ under $\mathrm{N}_{2}$ atmosphere for $2 \mathrm{~h}$ with a heating rate of $5{ }^{\circ} \mathrm{C} \mathrm{min}^{-1}$. During the formation of nanocomposite and high-temperature treatment, GO was partly reduced to rGO. Finally, a fine powder (the target sample) was obtained and collected for characterization and analysis. The process to synthesize the control group is displayed in the ESI. $\dagger$ Corresponding photographs of three samples solution are also provided in Fig. S1. $\dagger$

To investigate the effect of rGO amount on sensing activity, a series of $\mathrm{WO}_{3}-\mathrm{rGO}$ samples with different contents of rGO (about $4.1 \mathrm{wt} \%, 6.1 \mathrm{wt} \%, 7.9 \mathrm{wt} \%$ and $9.7 \mathrm{wt} \%$, respectively) was prepared with different rGO amounts by adjusting the addition of GO in solutions of $10 \mathrm{~mL}, 15 \mathrm{~mL}, 20 \mathrm{~mL}$ and $25 \mathrm{~mL}$, denoted as S1-10, S1-15, S1-20 and S1-25, respectively, while the other conditions remained the same.

\subsection{Characterization}

The X-ray diffraction (XRD) patterns of as-obtained sample were collected by a Rigaku TTR-III operated at $40 \mathrm{kV}$ and $150 \mathrm{~mA}$ with high-intensity $\mathrm{Cu}-\mathrm{K} \alpha$ radiation $(\lambda=0.15406 \mathrm{~nm})$. The morphology and micro-structure of samples were mainly investigated by a transmission electron microscope (FEITEM, Tecnai G220S-Twin) with an accelerating voltage of $120 \mathrm{kV}$ (HRTEM at $200 \mathrm{kV}$ ). Energy-dispersive X-ray spectrometry (EDX) was employed to research the elemental compositions of the 


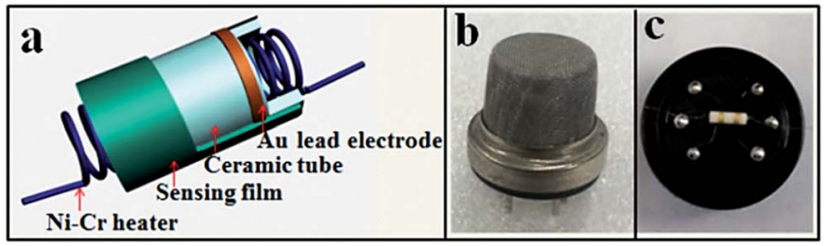

Fig. 1 (a) Schematic diagram of the gas sensor, (b, c) photograph of the sensor.

samples. Surface chemical analysis of simples was performed by X-ray photoelectron spectroscopy (XPS, PHI 5400 ESCA System) with an $\mathrm{Al}-\mathrm{K} \alpha$ radiation source. The specific surface areas were evaluated by the Brunauer-Emmett-Teller (BET) equation from a micrometrics TriStar II 3020 instrument at a liquid nitrogen temperature. Pore-size distribution was estimated using the Barrett-Joyner-Halenda (BJH) method from the desorption branch of isotherms. Sample was degassed under vacuum at $90^{\circ} \mathrm{C}$ for $3 \mathrm{~h}$ before gas tests.

\subsection{Fabrication and measurement of gas sensor}

The fabricating procedure, taking the sensor based on S1 as an example, was carried out following a similar process described in previous literature: ${ }^{31}$ a specific amount of S1 was dispersed with deionized water to form a homogeneous suspension with ultrasonic or continued grinding, and then uniformly coated onto a ceramic tube to form a sensing film (with a thickness of $c a .10 \mu \mathrm{m}$ ). Pt leads, used as electrical contacts, were attached to a pair of Au electrodes which were printed previously. After the calcined process (at $150{ }^{\circ} \mathrm{C}$ for $2 \mathrm{~h}$ to improve the reproducibility), a Ni-Cr alloy coil was inserted into the tube as a heater for tuning the operating temperature. The schematic diagram and the physical photograph of the sensor are shown in Fig. 1ac. The sensors were measured in a chamber with a volume of $10 \mathrm{~L}$ under laboratory conditions $\left(20 \% \mathrm{RH}, 25{ }^{\circ} \mathrm{C}\right)$. During the process, a moderate tested gas was injected into the chamber and diluted by fresh air to achieve a certain concentration. The gas sensing data were collected through a steady-state process conducted on a commercial NMDOG Multifunctional Precision Sensor Analysis Tester (Changsha Dingcheng Scientific Instrument Co., Ltd., Hunan, China). ${ }^{21,25,31}$

The gas response $(R)$ of the sensor is defined as $R=R_{\mathrm{g}} / R_{\mathrm{a}}$ for oxidizing gas or the reciprocal $\left(R_{\mathrm{a}} / R_{\mathrm{g}}\right)$ for reducing gas, where $R_{\mathrm{a}}$ and $R_{\mathrm{g}}$ are the measured resistances of the sensor in air and in the testing gas atmosphere, respectively. The response time and recovery time are defined as the time taken by the sensor to achieve $90 \%$ of the total resistance change after the sensor was exposed to the tested gas and air, respectively.

\section{Results and discussions}

\subsection{Structural and morphological characteristics}

To examine the crystal structure and phase composition, XRD patterns of the final target product $\left(\mathrm{WO}_{3}-\mathrm{rGO}\right)$, the standard card of $\mathrm{WO}_{3}$ (JCPDS no. 43-1035) and the original GO samples are depicted in Fig. 2. As displayed clearly, Fig. 2a presents the
XRD patterns of the as-obtained GO sample, from which a strong peak at $2 \theta=11.26^{\circ}$, corresponding to the (001) interlayer, indicates the successful preparation of GO by the oxidation of graphite. ${ }^{5,14} \mathrm{Fig} .2 \mathrm{~b}$ and $\mathrm{c}$ are the standard cards of $\mathrm{WO}_{3}$ (JCPDS no. 43-1035) and the target $\mathrm{WO}_{3}-\mathrm{rGO}$ composite. The XRD pattern in Fig. 2c confirms that the tungsten oxide in asobtained composite is monoclinic $\mathrm{WO}_{3}$ with lattice parameters: $a \times b \times c=7.297 \times 7.539 \times 7.688 \AA^{3}$ (space group: $P 21$ / $n(14)$ ), and which was indexed with a relevant crystallographic standard database structure (JCPDS no. 43-1035)..$^{25,26}$ In addition, the corresponding lattice planes (the $h k l$ values) are marked at the right diffraction angles. By comparison, most peaks of the black curve agree well with the reported values of monoclinic $\mathrm{WO}_{3}$ and no other crystalline peaks from impurity appear. More noticeably, the characteristic diffraction peak of GO is no longer observed which confirm that GO has transformed to a reduction state of $\mathrm{GO}(\mathrm{rGO}) \cdot{ }^{16}$ And coupled with the strong and sharp peaks, XRD results indicate that the $\mathrm{WO}_{3}-\mathrm{rGO}$ sample possess high purity and crystallinity. A similar analysis was carried out to $\mathrm{WO}_{3}$ lamellas (S2).

As confirmed, an optimized solution-based process is crucial to the synthesis composite with special architecture. ${ }^{4,30}$ During this experiment, benefit from feasible hydrothermal techniques, like high homogeneity at the molecular level, facile compositional control and mild solution system, we realize the successful preparation of mono-crystalline $\mathrm{WO}_{3}-\mathrm{rGO} .^{32}$ The formation of $\mathrm{WO}_{3}$ nanoflakes is attributed to the hydrolysis of $\mathrm{Na}_{2} \mathrm{WO}_{4}$ to $\mathrm{H}_{2} \mathrm{WO}_{4} \cdot 2 \mathrm{H}_{2} \mathrm{O}$ under weak acid buffer conditions. ${ }^{26} \mathrm{~A}$ simplified reaction is illustrated by the following formula:

$$
\begin{aligned}
& \mathrm{Na}_{2} \mathrm{WO}_{4}+2 \mathrm{H}^{+}+\underset{2}{2 \mathrm{H}_{2} \mathrm{O} \rightarrow} \\
& \mathrm{H}_{2} \mathrm{WO}_{4} \cdot 2 \mathrm{H}_{2} \mathrm{O} \text { (light yellow) } \downarrow+2 \mathrm{Na}^{+}
\end{aligned}
$$

On the surface of GO nanosheets, the presence of numerous functional groups, such as - $\mathrm{OH},-\mathrm{COO}-$ and epoxy, results in tight $\mathrm{C}-\mathrm{O}-\mathrm{W}$ bonding of $\mathrm{WO}_{3}$ nanosheets to $\mathrm{GO}$, which is confirmed by the following characterizations.

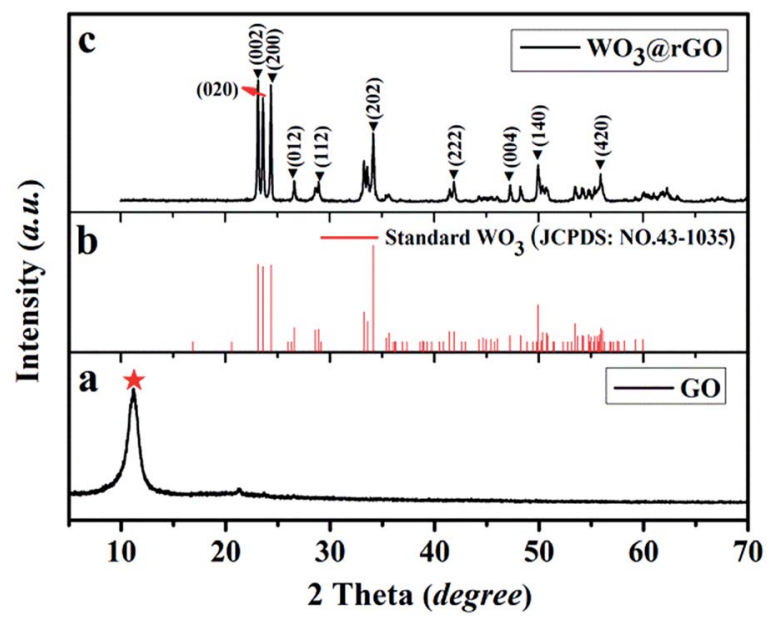

Fig. 2 XRD pattern of the as-obtained samples: (a) the GO, (b) JCPDS of $\mathrm{WO}_{3}$ and (c) the target $\mathrm{WO}_{3}-\mathrm{rGO}$ composite, S1. 
The morphological and structural features of the as-obtained samples were investigated in detail with corresponding TEM images shown in Fig. 3. A low-resolution TEM image of the asobtained $\mathrm{WO}_{3}-\mathrm{rGO}$ sample is displayed in Fig. 3a, from which paper-like rGO sheets of greater crinkling than GO (Fig. 3e) are observed. The rGO sheets present a nearly transparent and micrometre-long sheet-like structure which overlaps at the edges. Also, we see clearly that the dark-coloured $\mathrm{WO}_{3}$ nanoflakes embed into the plicated rGO sheets and grow together, presenting a quadrilateral morphology. As such, TEM observations are in disagreement with the XRD patterns, which reflect that the obtained $\mathrm{WO}_{3}$ flakes in the composite are not dominated by any crystallographic facets. As observed in TEM, the flake planes are not oriented parallel to one another, but inversely and irregularly arranged. We believe that the $\mathrm{WO}_{3}$ nanoflakes anchored onto or growing through the rGO sheets can greatly avoid the restacking process of rGO nanosheets. ${ }^{29}$ From Fig. 3b, the evident and well-ordered lattice fringe reveals that $\mathrm{WO}_{3}$ nanoflakes existing in the composite form a singlecrystalline structure. ${ }^{26}$ The fringe pattern indicates that a single crystalline flake has interplanar distances of 0.364 and $0.376 \mathrm{~nm}$, corresponding to the (200) and (020) crystal planes of the monoclinic $\mathrm{WO}_{3}$ phase, respectively. ${ }^{26,32}$ Impressively, the clear and distinct diffraction spots of the selected area electron diffraction (SAED) pattern (Fig. 3c) also confirm that the $\mathrm{WO}_{3}$ is of a single crystalline structure as well. ${ }^{26}$ Similarly, Fig. $3 \mathrm{~d}$ and e show the low-TEM images of the control groups S2 and S3, respectively. Fig. 3d presents the as-prepared $\mathrm{WO}_{3}$ nanolamellas with a uniform size of $500 \times 500 \mathrm{~nm}^{2}$ (length $\times$ width). We also notice that these nanolamellas are thin and have a rough and porous structure. In Fig. 3e, the TEM image of the original GO sheets is also given, from which we note that the sheet is also nearly transparent with many crinkles. Next, the HR-TEM image of $\mathrm{S} 2$ is shown in Fig. 3f, from which an enlarged image (inset) is

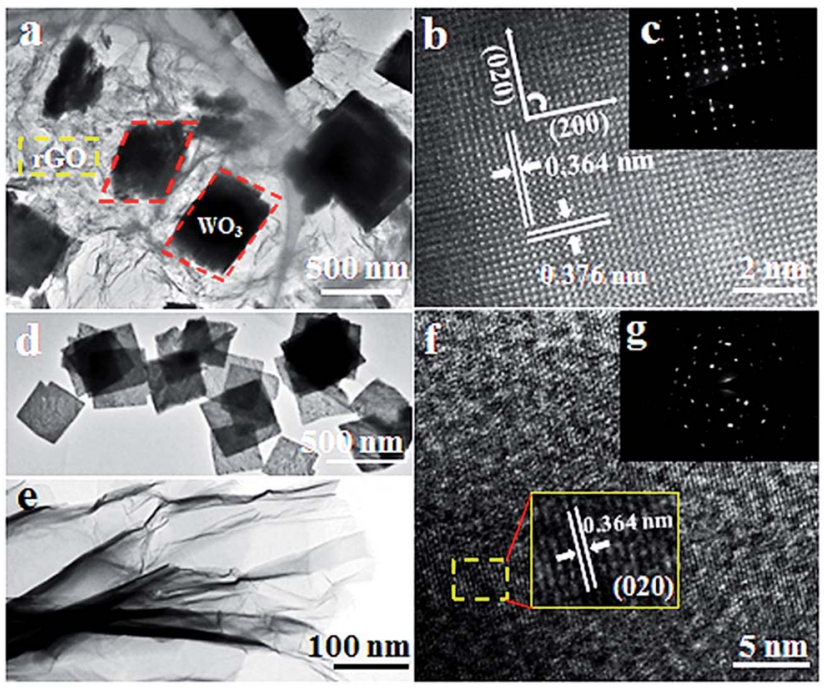

Fig. 3 TEM images: (a) low-resolution, (b) high-resolution and (c) SAED image of $\mathrm{WO}_{3}-\mathrm{rGO}$; (d) LR-image of $\mathrm{WO}_{3}$ nanolamellas, (e) LRimage of the original GO, (f) HR-image and (g) SAED of $\mathrm{WO}_{3}$ nanolamellas. taken. The distance between adjacent lattice planes is measured at $c a .0 .364 \mathrm{~nm}$, which matches well with the (200) plane of $\mathrm{WO}_{3}{ }^{27}$ And the corresponding SAED pattern is clearly shown in Fig. $3 g$ (the inset). The EDS element analysis of S1 in Fig. S3 $\dagger$ confirms that the hierarchical composite consists of $\mathrm{W}, \mathrm{O}$ and $\mathrm{C}$ elements.

To complete the previous analysis, XPS analysis reveals the surface chemical composition and electronic states of the elements of the $\mathrm{WO}_{3}-\mathrm{rGO}$ product (Fig. 4). All the binding energies obtained in the XPS spectra were calibrated for specimen charging by referencing to the $\mathrm{C}$ 1s peak (set at 284.60 eV). ${ }^{5,30}$ The full wide-scan spectrum of the as-obtained $\mathrm{WO}_{3}-$ rGO composite is presented in Fig. 4a, from which we observe clearly characteristic peaks of $\mathrm{W}, \mathrm{O}$ and $\mathrm{C}$ elements. The highresolution $\mathrm{W}$ 4f spectrum in Fig. $4 \mathrm{~b}$, displays two peaks with binding energy values of 37.98 and $35.83 \mathrm{eV}$ for $\mathrm{W} 4 \mathrm{f}_{5 / 2}$ and $\mathrm{W}$ $4 \mathrm{f}_{7 / 2}$ indicating the $\mathrm{W}(\mathrm{vI})$ oxidation state of $\mathrm{WO}_{3}-\mathrm{rGO}$ sample. .5,26,32 $^{2}$ The $\mathrm{O} 1 \mathrm{~s}$ peaks (Fig. 4c) at 530.1 and $532.0 \mathrm{eV}$ match well with oxygen species in the as-obtained composite. As acknowledged widely, the peak at $\sim 530.6 \mathrm{eV}$ can be assigned to typical surface lattice oxygen and the peak at $532.2 \mathrm{eV}$ is characteristic of surface adsorbed oxygen species., ${ }^{5,30,32}$ The surface adsorbed oxygen species play a positive role in the enhancement of gas sensing performances because they act as a key component to react with test gas molecules. Fig. $4 \mathrm{~d}$ shows the $\mathrm{C}$ 1s spectrum of $\mathrm{WO}_{3}-\mathrm{rGO}$, where three strong peaks at 284.6, 286.6 and $288.4 \mathrm{eV}$, are attributed to $\mathrm{C}-\mathrm{C}, \mathrm{C}-\mathrm{O}$ and $\mathrm{C}=\mathrm{O}$ bands in graphene-based materials, respectively. ${ }^{5,14,29}$ To further confirm and identify the composition, especially rGO, we carried out FT-IR analysis which reveals FT-IR absorption spectra of $\mathrm{WO}_{3}, \mathrm{GO}$ and $\mathrm{WO}_{3}-\mathrm{rGO}$ composite (Fig. 5). As shown in green (Fig. 5a), the most obvious absorption peak at 3420 $\mathrm{cm}^{-1}$ of high frequency is ascribed to the stretching vibration of $-\mathrm{O}-\mathrm{H} .{ }^{14,33}$ And as reported, the absorption band at $1636 \mathrm{~cm}^{-1}$ is ascribed to the skeletal vibration of GO sheets. ${ }^{6,34}$ Absorption bands at 1732, 1386 and $1078 \mathrm{~cm}^{-1}$ are ascribed to a typical stretching vibration of $-\mathrm{C}=\mathrm{O}$ (carboxyl), deformation vibration
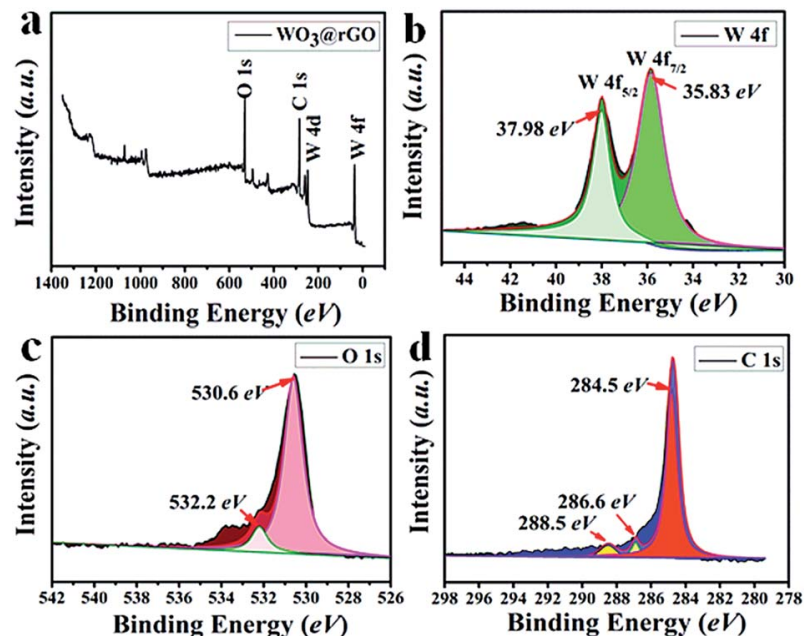

Fig. 4 XPS spectra of as-obtained $\mathrm{WO}_{3}-\mathrm{rGO}$ composite: (a) survey scan; high-resolution (b) W 4f, (c) $O 1 \mathrm{~s}$, and (d) $C 1$ s spectrum. 


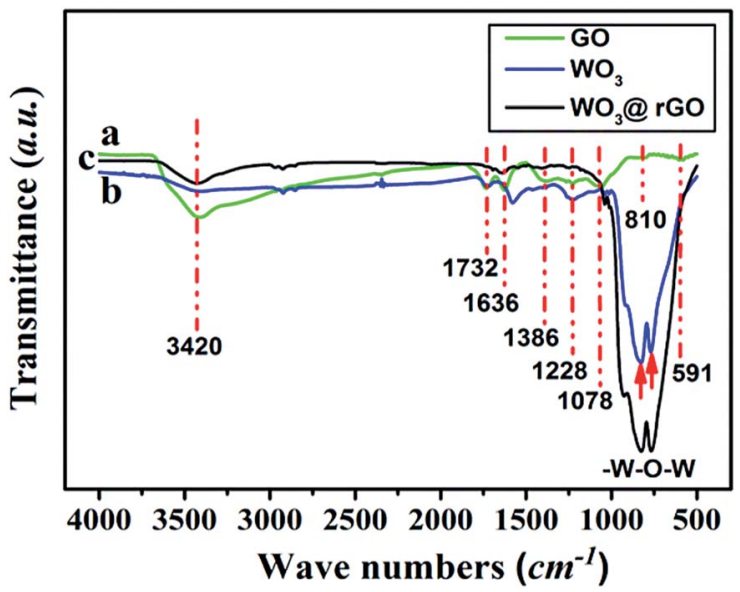

Fig. 5 FT-IR spectra of the as-obtained $\mathrm{GO}$ ( $\mathrm{a}$, in green), $\mathrm{WO}_{3}$ (b, in blue) and $\mathrm{WO}_{3}-\mathrm{rGO}$ (c, in black).

of $-\mathrm{O}-\mathrm{H}$ and stretching vibration of $-\mathrm{C}-\mathrm{O}$ (alkoxy), respectively. ${ }^{5,26}$ The weak absorption peaks at around 810 and 591 $\mathrm{cm}^{-1}$ are due to the epoxide groups situated at the edges of the GO sheets. ${ }^{21,35}$ As for the target $\mathrm{WO}_{3}-\mathrm{rGO}$ composite (in black, Fig. 5c), absorption bands at 1732, 1386 and $1078 \mathrm{~cm}^{-1}$ weaken distinctly, suggesting that reduced GO forms during the synthetic process. ${ }^{17}$ Besides, as for the $\mathrm{WO}_{3}-\mathrm{rGO}$ composite, absorption bands at low frequencies (below $1000 \mathrm{~cm}^{-1}$ ) are ascribed to the vibration of $\mathrm{W}-\mathrm{O}-\mathrm{W}$ of crystalline $\mathrm{WO}_{3}$, which are not observed in the spectrum of GO but appear in $\mathrm{WO}_{3}$ (in blue, Fig. 5b). ${ }^{29}$ Compared with GO, the peak intensity of oxygen-containing functional groups (mainly $-\mathrm{OH},-\mathrm{C}-\mathrm{O}$ and $-\mathrm{C}=\mathrm{O}$ ) weakens distinctly in the spectrum of $\mathrm{WO}_{3}-\mathrm{rGO}$ composite, which is caused by the formation of nanocomposite and high-temperature annealing process. ${ }^{17,29,33}$ Accordingly, upon hydrothermal treatment, $\mathrm{GO}$ is reduced to $\mathrm{rGO}$ gradually and is accompanied by the formation of $\mathrm{C}-\mathrm{O}-\mathrm{W}$ bond. The annealing process is also carried out to promote the reduction of GO by losing oxygen-containing functional groups. ${ }^{27}$ Additionally, no other evident absorption peaks are observed from the $\mathrm{WO}_{3}-\mathrm{rGO}$ sample, indicating that no new bonding forms between the $\mathrm{WO}_{3}$ and rGO matrix. Combined with the XRD pattern, these observations above confirm the successful preparation of $\mathrm{WO}_{3}-\mathrm{rGO}$ sheet-like composite.

The surface structural characteristics of the as-obtained three samples were further analysed by $\mathrm{N}_{2}$ adsorption-desorption techniques. The corresponding BET data are displayed in Fig. S4 $\uparrow$ and summarized in Table 1 . As shown in Fig. S4a, $\dagger$ the prepared sample exhibits a surface area of $102.3 \mathrm{~m}^{2} \mathrm{~g}^{-1}$ and the isotherm profile is categorized as type IV with a small hysteresis loop, which indicates that a porous structure (mesopores and micropores) exists as part of the hierarchical structure. ${ }^{30,32,36}$ The pore-size distribution (inset, $\mathrm{S} 4 \mathrm{~b} \dagger$ ) was determined by $\mathrm{BJH}$ method from the desorption branch of the isotherm, from which we observe that the average pore diameter of the sample is $15-40 \mathrm{~nm}$ (mesoporous, mainly). These favourable porous structures not only contribute to the increase of specific surface area $(\mathrm{S} 1>\mathrm{S} 2>\mathrm{S} 3)$ and active sites, but also to an increase of
Table 1 Comparisons on the BET data and response to $\mathrm{NO}_{2}$ of three samples $^{a}$

\begin{tabular}{llll}
\hline Number & Materials & $\begin{array}{l}\text { BET surface } \\
\text { area } /\left(\mathrm{m}^{2} \mathrm{~g}^{-1}\right)\end{array}$ & $R$ \\
\hline $\mathrm{S} 1$ & $\mathrm{WO}_{3}-\mathrm{rGO}$ & 102.3 & $\sim 4.3$ \\
$\mathrm{~S} 2$ & $\mathrm{WO}_{3}$ & 36.2 & $\sim 1.8$ \\
$\mathrm{~S} 3$ & $\mathrm{GO}$ & 24.5 & $\sim 1.1$ \\
${ }^{a} R$, response at $90{ }^{\circ} \mathrm{C}$. & & \\
& & &
\end{tabular}

transfer channels for gas diffusion. All the BET data are consistent with the observed results of TEM images. The BET data of other samples (S2, S3) are shown in Fig. S4 $\dagger$ and are summarized in Table 1.

\subsection{Gas sensing properties}

To highlight the advantages in the gas sensing area of these interesting structures, gas sensors based on the as-prepared products were fabricated to evaluate their sensing properties. Operating temperature is one of the most significant parameters for a successful gas sensor. As known, the whole sensing process is intimately associated with the variation of temperature, which influences gas diffusion, gas adsorption/desorption and crucial sensing reactions. Therefore, parallel experiments were carried out to determine the optimum operating temperature, ranging from $20{ }^{\circ} \mathrm{C}$ to $200{ }^{\circ} \mathrm{C}$, while exposing the sensor to $10 \mathrm{ppm}$ of ambient $\mathrm{NO}_{2}$. The test responses toward $10 \mathrm{ppm}$ $\mathrm{NO}_{2}$ at different temperatures are summarized in Fig. 6. We notice that the response values of tested sensors vary dramatically with operating temperature. Namely, the responses all increase with operating temperature at the initial phase and reach a maximum value at a specific temperature. After achieving the maximum, a distinct decline is observed, confirming the existence of optimal temperature. Fig. 6 demonstrates that the optimal working temperature of $\mathrm{WO}_{3}-\mathrm{rGO}$ composite appears at $90^{\circ} \mathrm{C}$, while pure $\mathrm{WO}_{3}$ 's optimal working temperature is $140{ }^{\circ} \mathrm{C}$. In detail, the response values at $90^{\circ} \mathrm{C}$ are

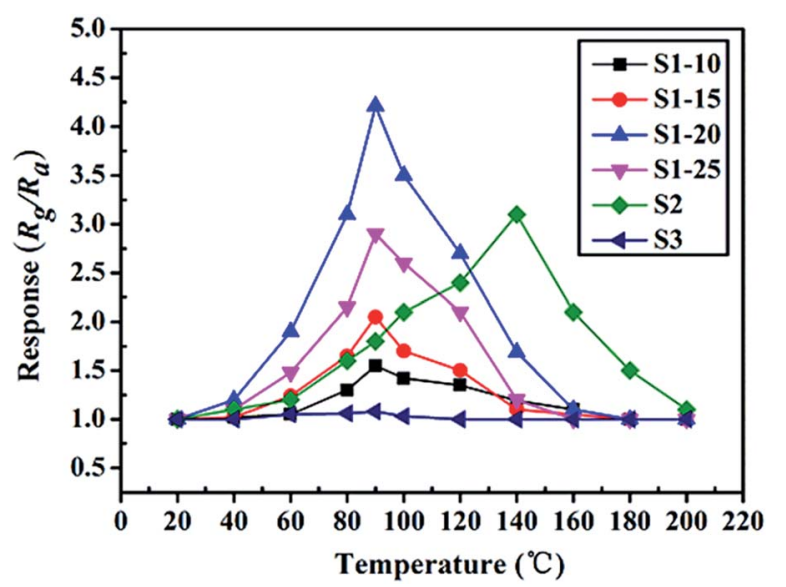

Fig. $6 \mathrm{NO}_{2}$ gas sensing property of the sensors at different working temperatures. 
1.6, 2.1, 4.3, 2.9, 1.8 and 1.1 for S1-10, S1-15, S1-20, S1-25, S2 and S3 toward $\mathrm{NO}_{2}$, respectively. We can notice that the composite enhances gas sensing response, especially with respect to working temperature, which is comparable to recent reports. ${ }^{27,33,37}$ Also, the result indicates that the amount of rGO in the composite has an important role in $\mathrm{NO}_{2}$ detection. By comparison, the responses of S1-20 sensor are superior to other sensors based on composites with other contents. We also provide the gas sensing results of the original GO sample, which has a weak sensing response to $\mathrm{NO}_{2}$ in the temperature range 20-220 ${ }^{\circ} \mathrm{C}$. To illustrate the advantages of the composite, we summarize the response results of $\mathrm{S} 1$ and $\mathrm{S} 2$ with a bar graph in Fig. 7a. Based on repeated trials, the response of porous $\mathrm{WO}_{3}-$ rGO composite achieves a peak value of 4.3 , which is almost fourfold higher than the control group (S2) at $90{ }^{\circ} \mathrm{C}$. The significant enhancement in the response of $\mathrm{S} 1$ toward $\mathrm{NO}_{2}$ is mainly ascribed to the successful attachment of $\mathrm{WO}_{3}$ nanosheets onto rGO to form a 3-D $\mathrm{WO}_{3}-\mathrm{rGO}$ hybrid nanostructure. Besides, the favorable morphology and porous structure in the composite provides many channels for gas diffusion.

Selectivity is an important parameter of gas sensors for their practical application; the gas response toward a specific gas needs to be markedly higher than for other gases. To explore the selectivity of S1, a bar graph (Fig. 7b) of the sensor based on asobtained $\mathrm{WO}_{3}-\mathrm{rGO}$ toward several different gases like methanol, ethanol, acetone, $\mathrm{NO}_{2}$ and $\mathrm{H}_{2} \mathrm{~S}$, is displayed. All gases were tested at $90{ }^{\circ} \mathrm{C}$ under same concentration (10 ppm). As shown, the sensor possesses a good selectivity to $\mathrm{NO}_{2}$ gas in comparison to other tested gases. It is worth noting that the responses to methanol $(\sim 1.4)$, ethanol $(\sim 1.1)$, toluene $(\sim 1)$ and xylene $(\sim 1)$ are minor and can be ignored, when compared to $\mathrm{NO}_{2}$.

Next, the dynamic response transients' features of $\mathrm{S} 1$ at $90{ }^{\circ} \mathrm{C}$ are summarized in Fig. 8a to highlight the monotonicity of response versus gas concentration. Clearly, the response value increases with the increase in gas concentration, indicating that the sensor (S1) responds well to this variation. Besides, it is noteworthy that at a lower concentration range the response is more acute. As mentioned, the response value of a sensor mainly depends on the surface chemical reaction. Hence, when the concentration of the test gas is relatively low, there are fewer reactions occurring on the surface. Along with the increase in concentration, the sensing reaction increases sharply, resulting in a linear trend. However, when the concentration exceeds
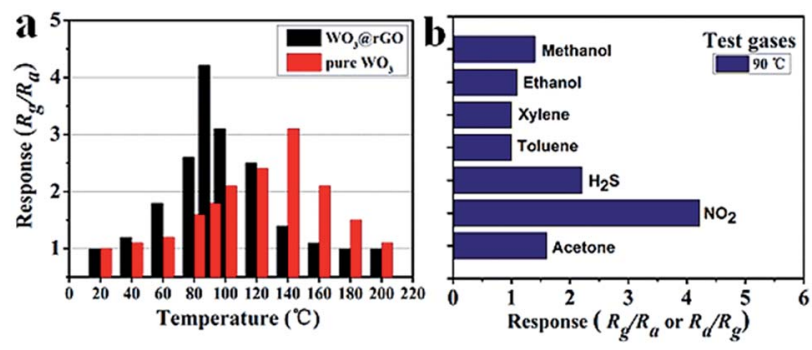

Fig. 7 (a) Response of S1 and S2 versus operating temperature to $10 \mathrm{ppm} \mathrm{NO} \mathrm{N}_{2}$, (b) response of sensor based on S1 to $10 \mathrm{ppm}$ various gases at $90^{\circ} \mathrm{C}$.
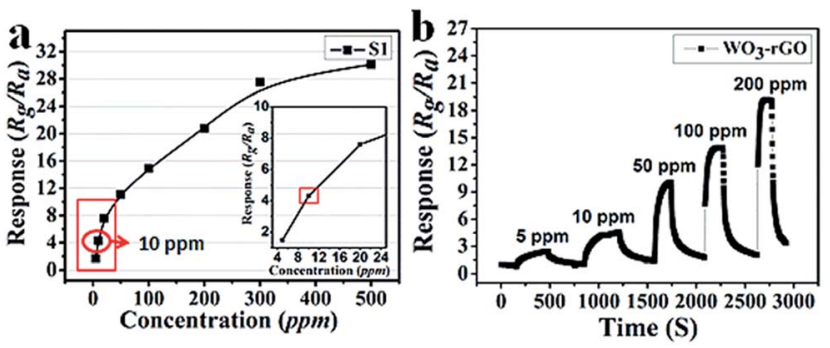

Fig. 8 (a) The relationship plots of response versus concentration and (b) dynamic response transients of $\mathrm{S} 1$ sample to 5-200 ppm NO 2 at $90{ }^{\circ} \mathrm{C}$.

200 ppm and continues rising, this trend levels off because most active sites have already reached saturation. In Fig. 8b, the dynamic response transients of $\mathrm{S} 1$ present nearly a square shape, which suggests that when the sensor is exposed to $\mathrm{NO}_{2}$ gas, the response value achieves a maximum quickly. The maintained state can also be explained by saturated reaction sites not causing effective resistance variation or changing slightly. While the sensor is exposed to fresh air, the response returns to a near baseline level.

The response and recovery times also play a vital part in the practical detection to some detrimental and dangerous gases. ${ }^{21,34}$ In theory, fast charge separation occurs at the interface of the heterostructure and the body of sensing material, so that the generated electrons can efficiently react with test gases during the sensing process and bring about an efficient response/ recovery speed..$^{\mathbf{6} 34,38}$ Besides, a favourable nano/micro-structure also benefits gas diffusion and mass transport, and improvements in response/recovery time, such as a porous and surfaceroughened sheet-like structure. To evaluate our sensing material systematically, Fig. 9 shows the response and recovery times of sensors toward $10 \mathrm{ppm} \mathrm{NO}$. By contrast, the response times are 4.1 and $10.3 \mathrm{~s}$ for $\mathrm{S} 1$ and $\mathrm{S} 2$, while the corresponding recovery times are 5.8 and $8.7 \mathrm{~s}$, respectively. The long-term cyclic stability of the sensor based on S1 composite was measured (Fig. 10). From Fig. 10a the sensor exhibits nearly constant sensing signals in one month and quite similar shapes at each time to $10 \mathrm{ppm}$ $\mathrm{NO}_{2}$ during the test (Fig. 10b), confirming its improved stability and repeatable performance in application.
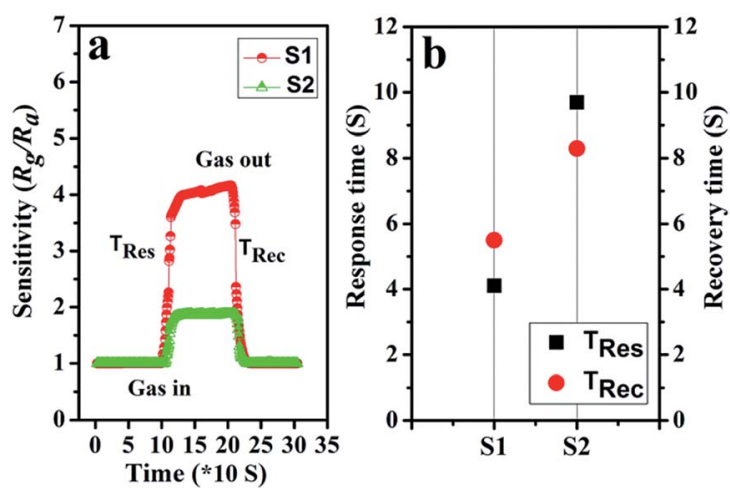

Fig. 9 (a) The dynamic $\mathrm{NO}_{2}$ response transients of $\mathrm{S} 1$ and $\mathrm{S} 2$ at $90{ }^{\circ} \mathrm{C}$; (b) the plot of response/recovery time of sensors. 


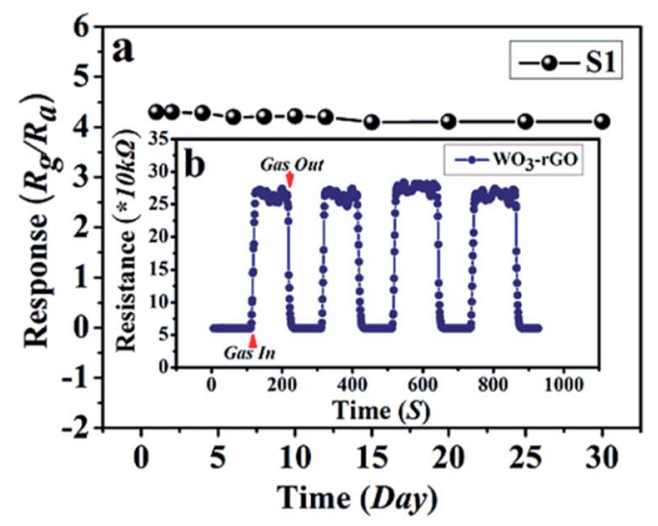

Fig. 10 (a) Long-term stability of gas sensor based on S1 to $10 \mathrm{ppm}$ $\mathrm{NO}_{2}$ at $90{ }^{\circ} \mathrm{C}$; (b, the inset) the dynamic resistance transients of $\mathrm{S} 1$ to $10 \mathrm{ppm} \mathrm{NO} \mathrm{N}_{2}$ during the stability test.

\subsection{Gas sensing mechanism}

Gas sensors based on binary composite have attracted a lot of attention owing to their excellent sensing performance. As reported in previous literature, a popular and widely accepted gas sensing mechanism model was employed to illustrate the sensing process of the target $\mathrm{WO}_{3}-\mathrm{rGO}$ composite, namely surface-controlled model. ${ }^{4,14,30,36}$

In this case, the resistance of sensor produces regular signal variations caused by the adsorption, reaction and desorption processes of gas molecules on the surface/interface. When the sensor is exposed to air, oxygen molecules in air will be chemisorbed and dissociates on the surface of the sensing film. These $\mathrm{O}_{2}$ molecules will capture electrons, transferring them from a conduction band to chemisorbed oxygen and generating ion-adsorbed oxygen species $\left(\mathrm{O}_{2}{ }^{-}, \mathrm{O}^{-}, \mathrm{O}^{2-}\right)$ film on the surface of the sensing layer. This results in a decrease in carrier density and formation of an electron depletion region (EDR) near the surface of sensing materials, leading to an initial resistance. ${ }^{29,39-41}$ The specific reactions are as follows (eqn (1)-(3)):

$$
\begin{gathered}
\mathrm{O}_{2}(\text { gas }) \rightarrow \mathrm{O}_{2} \text { (ads) } \\
\mathrm{O}_{2}(\text { ads })+\mathrm{e}^{-} \rightarrow \mathrm{O}_{2}^{-} \text {(ads) } \\
\mathrm{O}_{2}^{-} \text {(ads) }+\mathrm{e}^{-} \rightarrow 2 \mathrm{O}^{-} \text {(ads) }
\end{gathered}
$$

On exposure to $\mathrm{NO}_{2}$ (target gas), the $\mathrm{NO}_{2}$ as an oxidizing gas with relatively strong electrophilic property, ${ }^{37}$ seizes electrons from the energy band of the sensing film and pre-adsorbed oxygen ions as in the reactions below:

$$
\begin{gathered}
\mathrm{NO}_{2} \text { (ads) }+\mathrm{e}^{-} \rightarrow 2 \mathrm{NO}_{2}{ }^{-} \\
\mathrm{NO}_{2} \text { (gas) }+\mathrm{O}_{2}{ }^{-}+2 \mathrm{e}^{-} \rightarrow \mathrm{NO}_{2}{ }^{-}+2 \mathrm{O}^{-} \text {(ads) }
\end{gathered}
$$

These eqn (4) and (5) indicate that the total resistance increases with the injection of $\mathrm{NO}_{2}$ gas, leading to a significant increase in the sensor's resistance. When $\mathrm{NO}_{2}$ supply is cut off, the gas sensing reaction starts on the recovery stage and the adsorbed $\mathrm{NO}_{2}{ }^{-}$ions begin to desorb. This process will release electrons trapped by adsorbed $\mathrm{NO}_{2}{ }^{-}$and $\mathrm{O}^{-}$ions back into the conduction band, forming an electron accumulation region (EAR) near the surface. ${ }^{27,32}$ Thus, the resistance will recover to its initial value, and generate another electrical signal. Eventually, a gas sensing cycle is obtained. The reaction in this stage is simply described by eqn (6):

$$
\mathrm{NO}_{2}{ }^{-}(\mathrm{ads})+2 \mathrm{O}^{-}(\mathrm{ads}) \rightarrow \mathrm{NO}_{2}(\mathrm{gas})+\mathrm{O}_{2}(\text { gas })+2 \mathrm{e}^{-}(\text {ads })
$$

As the sensing test indicates, the whole of the $\mathrm{WO}_{3}-\mathrm{rGO}$ composite presents p-typed gas sensing behaviour. During the process, it means that p-type metal oxides only chemisorb as much oxygen as possible to compensate for their deficiencies, ${ }^{42,43}$ if the number of active sites and concentration of surface oxygen on p-type materials are not limited.

As confirmed, a gas sensor based on $\mathrm{WO}_{3}-\mathrm{rGO}$ sheet-like composite exhibits much enhanced response properties, compared with that of pure $\mathrm{WO}_{3}$ and GO. The excellent gas sensing performance mainly results from the following factors: first, compared to the test data of pristine GO nanosheets, the co-presence of $\mathrm{WO}_{3}$ and rGO have an intimate contact at the nano-level, which further contributes to the improvement of the whole sensing performance, especially in regard to response; second, the well-known electrochemical activity of rGO nanosheets is believed to play a further beneficial role on the functional behaviour. ${ }^{14}$ The outstanding conductivity of rGO supported material dramatically improves electron transfer efficiency of the composite, embodied in the enhancement of the sensor's response/recovery speed. And as reported, the products, $\mathrm{WO}_{3}$ and $\mathrm{rGO}$, both possess better sensing properties toward the test $\mathrm{NO}_{2}$ gas. ${ }^{26,32,33}$ The synergistic effect is also employed to explain the enhancement of composite's properties. ${ }^{26-29}$ Besides, the interesting 3-D structural characteristics, loose and porous structure, and large available surface area, are crucially important to shorten the response and recovery times, and to enhance response of the gas sensor.

\section{Conclusions}

In summary, a facile and cost-effective hydrothermal synthesis method was successfully employed to prepare 3-D $\mathrm{WO}_{3}-\mathrm{rGO}$ composite. The novel structure of as-obtained sample was characterized by a series of analytic techniques, and its gas sensing properties were investigated at length. We found that the introduction of $\mathrm{WO}_{3}$ into rGO nanosheets significantly enhanced the whole $\mathrm{NO}_{2}$ sensing performance, reflecting in low working temperature, responding effectively and having a wide linear range and good selectivity to $\mathrm{NO}_{2}$. The reasons for this phenomenon are discussed and explained by the formation of hetero-junctions, good electron transporting properties, and porous and ordered sheet-shaped structure. We believe that hierarchical $\mathrm{WO}_{3}-\mathrm{rGO}$ composite will have a great application not only in the gas-sensing field but also in other advanced functional material frontiers. We anticipate our work here provides more incentive to support the preparation of highperformance graphene-supported material in related areas. 


\section{Acknowledgements}

The author thanks the financial supports of the National Natural Science Foundation of China (51402065 \& 61473095), Fundamental Research Funds of the Central University (HEUCFZ), the Major Project of Science and Technology of Heilongjiang Province (GA14A101), International Science \& Technology Cooperation Program of China (2015DFR50050) and Natural Science Foundation of Heilongjiang Province (B201404).

\section{Notes and references}

1 R. A. Potyrailo, C. Surman, N. Nagraj and A. Burns, Chem. Rev., 2011, 111, 7315-7354.

2 R. Sui and P. Charpentier, Chem. Rev., 2012, 112, 3057-3082. 3 Y. Xu, L. Chen, X. Wang, W. Yao and Q. Zhang, Nanoscale, 2015, 7, 10559-10583.

4 J. H. Lee, Sens. Actuators, B, 2009, 140, 319-336.

5 S. Xu, F. Sun, Z. Pan, C. Huang, S. Yang, J. Long and Y. Chen, ACS Appl. Mater. Interfaces, 2016, 8, 3428-3437.

6 Y. Yang, C. Tian, J. Wang, L. Sun, K. Shi, W. Zhou and H. Fu, Nanoscale, 2014, 6, 7369-7378.

7 X. An, J. C. Yu, Y. Wang, Y. Hu, X. Yu and G. Zhang, J. Mater. Chem., 2012, 22, 8525.

8 Y. Du, X. Zhou, X. Zhou, L. Hu, Z. Dai and J. Bao, J. Mater. Chem. A, 2015, 3, 6787-6791.

9 S. Zhang, H. Liu, C. Huang, G. Cui and Y. Li, Chem. Commun., 2015, 51, 1834-1837.

10 Z. Gao, W. Yang, J. Wang, N. Song and X. Li, Nano Energy, 2015, 13, 306-317.

11 C. Wu, X. Lu, L. Peng, K. Xu, X. Peng, J. Huang, G. Yu and Y. Xie, Nat. Commun., 2013, 4, 2431.

12 G. Wang, L. Zhang and J. Zhang, Chem. Soc. Rev., 2012, 41, 797-828.

13 Y. Zhai, Y. Dou, D. Zhao, P. F. Fulvio, R. T. Mayes and S. Dai, Adv. Mater., 2011, 23, 4828-4850.

14 H. Zhang, J. Feng, T. Fei, S. Liu and T. Zhang, Sens. Actuators, $B, 2014,190,472-478$.

15 A. Cagliani, D. M. A. Mackenzie, L. K. Tschammer, F. Pizzocchero, K. Almdal and P. Boggild, Nano Res., 2014, 7, 743-754.

16 D. Zhang, H. Chang, P. Li, R. Liu and Q. Xue, Sens. Actuators, $B, 2016,225,233-240$.

17 Y. Xiao, Q. Yang, Z. Wang, R. Zhang, Y. Gao, P. Sun, Y. Sun and G. Lu, Sens. Actuators, B, 2016, 227, 419-426.

18 A. Lipatov, A. Varezhnikov, P. Wilson, V. Sysoev, A. Kolmakov and A. Sinitskii, Nanoscale, 2013, 5, 5426-5434.

19 S. Wang, X. Gao, J. Yang, Z. Zhu, H. Zhang and Y. Wang, RSC Adv., 2014, 4, 57967-57974.

20 J. Li, Q. Li, Y. Zeng, T. Tang, Y. Pan and L. Li, RSC Adv., 2015, 5, 717-725.
21 D. Guo, P. Cai, J. Sun, W. He, X. Wu, T. Zhang, X. Wang and X. Zhang, Carbon, 2016, 99, 571-578.

22 Z. Zhang, X. Zou, L. Xu, L. Liao, W. Liu, J. Ho, X. Xiao, C. Jiang and J. Li, Nanoscale, 2015, 7, 10078-10084.

23 G. Lu, L. E. Ocola and J. Chen, Adv. Mater., 2009, 21, 24872491.

24 Y. Liu, J. Parisi, X. Sun and Y. Lei, J. Mater. Chem. A, 2014, 2, 9919-9943.

25 S. Srivastava, K. Jain, V. N. Singh, S. Singh, N. Vijayan, N. Dilawar, G. Gupta and T. D. Senguttuvan, Nanotechnology, 2012, 23, 205501.

26 T. Kida, A. Nishiyama, Z. Hua, K. Suematsu, M. Yuasa and K. Shimanoe, Langmuir, 2014, 30, 2571-2579.

27 P. Su and S. Peng, Talanta, 2015, 132, 398-405.

28 V. K. Tomer, S. Devi, R. Malik, S. P. Nehra and S. Duhan, Sens. Actuators, B, 2016, 229, 321-330.

29 J. Shi, Z. Cheng, L. Gao, Y. Zhang, J. Xu and H. Zhao, Sens. Actuators, B, 2016, 230, 736-745.

30 X. Jie, D. Zeng, J. Zhang, K. Xu, J. Wu, B. Zhu and C. Xie, Sens. Actuators, B, 2015, 220, 201-209.

31 T. Liu, J. Liu, Q. Liu, D. Song, H. Zhang, H. Zhang and J. Wang, Nanoscale, 2015, 7, 19714-19721.

32 L. Hu, B. Qu, C. Li, Y. Chen, L. Mei, D. Lei, L. Chen, Q. Li and T. Wang, J. Mater. Chem. A, 2013, 1, 5596-5602.

33 M. Bao, Y. Chen, F. Li, J. Ma, T. Lv, Y. Tang, L. Chen, Z. Xu and T. Wang, Nanoscale, 2014, 6, 4063-4066.

34 D. Yang, K. Kang, D. Kim, Z. Li and I. Park, Sci. Rep., 2015, 5, 8149.

35 Y. Feng, N. Feng, G. Zhang and G. Du, CrystEngComm, 2014, 16, 214-222.

36 F. Qu, J. Liu, Y. Wang, S. Wen, Y. Chen, X. Li and S. Ruan, Sens. Actuators, B, 2014, 199, 346-353.

37 U. Yaqoob, A. S. M. I. Uddin and G. S. Chung, Sens. Actuators, $B, 2016,224,738-746$.

38 Y. Tian, J. Li, H. Xiong and J. Dai, Appl. Surf. Sci., 2012, 258, 8431-8438.

39 A. K. Nayak, R. Ghosh, S. Santra, P. K. Guha and D. Pradhan, Nanoscale, 2015, 7, 12460-12473.

40 D. Sarkar, X. Xie, J. Kang, H. Zhang, W. Liu, J. Navarrete, M. Moskovits and K. Banerjee, Nano Lett., 2015, 15, 28522862.

41 T. Liu, J. Liu, Q. Liu, Y. Sun, X. Jing, H. Zhang and J. Wang, CrystEngComm, 2016, 18, 5728-5735.

42 P. A. Russo, N. Donato, S. G. Leonardi, S. Baek, D. E. Conte, G. Neri and N. Pinna, Angew. Chem., Int. Ed., 2012, 51, 1105311057.

43 S. Cao, W. Zeng, H. Long and H. Zhang, Mater. Lett., 2015, 159, 385-388. 